\title{
Synthesis of Novel Iminosugar Derivatives based on a 2-Aza- bicyclo[4.1.0]heptane Skeleton
}

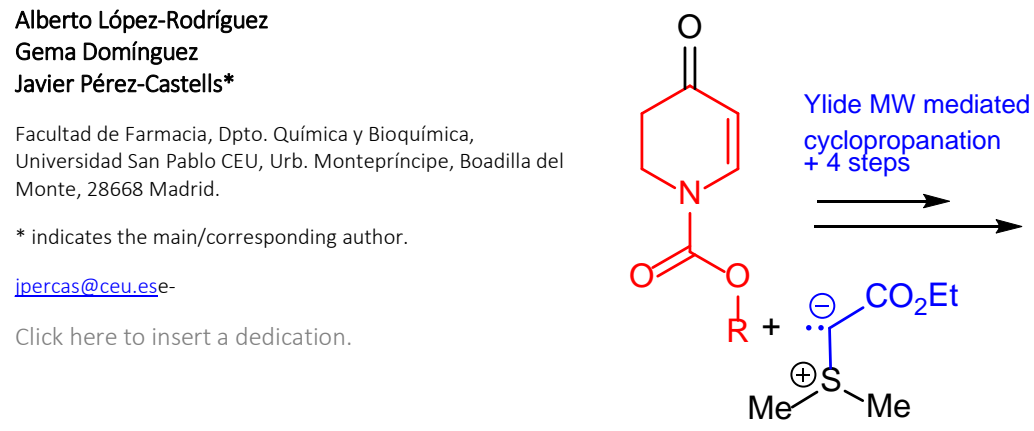

Alberto López-Rodríguez

Gema Domínguez

erez-Castells*

Facultad de Farmacia, Dpto. Química y Bioquímica,

Monte, 28668 Madrid.

* indicates the main/corresponding author.

jpercas@ceu.ese-

Click here to insert a dedication

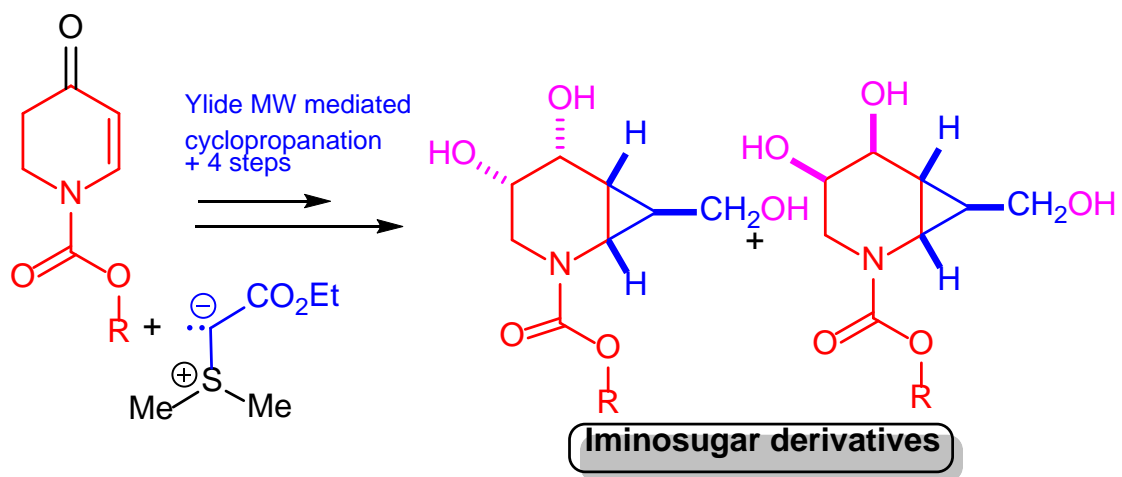

\begin{abstract}
Received:
Accepted: Publis
DOI:

Abstract: Iminosugars are good starting points for the development of different kinds of drugs. Many are polyhydroxylated piperidines that behave as biomimetics of their corresponding pyranoses analogs. In the interaction with carbohydrate processing enzymes, selectivity is a crucial issue and we have envisioned the benefits of introducing a cyclopropane bridge in a piperidine structure. We show the synthesis of novel bicyclic piperidine-based iminosugars using a sulfur ylide cyclopropanation as the key synthetic step.

Key words bicyclic compounds; piperidines; stereoselective synthesis; ylides; iminosugars; cyclopropanation
\end{abstract}

Glycosidases play a crucial role in many important biological processes. ${ }^{1}$ The design and synthesis of new glycosidase inhibitors opens the possibility of finding new therapeutic targets for the treatment of diabetes, AIDS, or cancer. ${ }^{2}$ In pursuing this goal, adequate metabolic stability and conformational mimicking of monosaccharides is needed as well as achieving enough selectivity towards specific glycosidases. Iminosugars are a family of natural and synthetic azaheterocycles with great similarity to carbohydrates, thus being able to compete with carbohydrates for the active site of glycosidases. ${ }^{3}$ They share with sugars the presence of various stereogenic centers so that they can be highly specific and may be useful in modulating the activity of several glycosidases. The replacement of the oxygen in the ring by a nitrogen maintains their absorption and cellular transportation mechanism. In addition, this basic nitrogen is protonated at physiological $\mathrm{pH}$ and mimics the intermediate formed during the hydrolysis of the glycosidic bond and various hydroxyl groups. Many iminosugars introduce a conformationally restricting motif that adds selectivity to a particular enzyme. Up to now, several competitive inhibitors of these enzymes are being developed as new drugs. Miglitol (Glyset ()$^{4}$ and miglustat (Zavesca $\left({ }^{\circledR}\right), 5$ for instance, are being commercialized for the treatment of diabetes type II and as the first oral treatment for Gaucher disease, respectively.

Many groups have studied the synthesis of iminosugars with a great emphasis on the preparation of piperidine derivatives. ${ }^{6}$ Many of these polyhydroxylated piperidine derivatives are related to natural iminosugar nojirimycin, and its 1deoxyanalogs deoxynojirimycins, which were isolated and characterized from natural sources. ${ }^{7}$ Lack of the 1-hydroxy group in the latter improves their stability in the biological environment. Different stereoisomers of deoxynojirimycin, are powerful inhibitors of glycosidases. Many derivatives and analogues of this family have also been synthesized. ${ }^{8}$

As a novel approach to improve selectivity of iminosugras we envisioned the possibility of rigidifying the piperidine structure by including a cyclopropanic bridge. ${ }^{9}$ We have designed new bicyclic iminosugars that include the cyclopropane motif aimed to fix the conformation, and hopefully improve the biological properties in terms of activity and selectivity. The resulting molecules have a flattened-chair conformation as a result of the fusion with the cyclopropane ring. The only previous work in which a cyclopropane ring was included in the structure was disclosed by Shipman. ${ }^{10}$ In addition, very recently, derivatives of iminosugars possessing carbamate groups have been described. ${ }^{11}$ Carbamates are relevant pharmacophores present for instance in antibiotics active against Gram-positive bacteria. ${ }^{12}$ Thus we have introduced in our structures two 
different carbamate groups, that may contribute to give interesting biological properties to the new compounds, or could be eliminated readily in further stages.

In this work we will present the synthesis of these new bicyclic iminosugars. The key cyclopropanation step and subsequent functional group modifications will give us different stereoisomers of the desired products.

The first step was the synthesis of enaminones 1a-b. These enaminones are well known building blocks that have received wide synthetic attention. ${ }^{13}$ We selected the procedure described by Minnaard and Feringa to transform 4-methoxypyridine into 1a-b although we used $\mathrm{NaBH}_{4}$ as the reducing agent. ${ }^{14}$ Similar yields as in the literature were achieved. The next step was the sulfur ylid mediated cyclopropanation, which was carried out using our recently disclosed procedure under microwave heating. This method allows to shorten the generally long reaction times used for these transformations. ${ }^{15}$ Thus, after the reaction of 1a-b with (2-ethoxy-2-oxoethyl)dimethylsulfonium bromide using DBU as the base the resulting products were reduced with $\mathrm{NaBH}_{4}$ which gave racemic 2a-b in 57-52 \% yield respectively after the two steps. Interestingly these two products were obtained as a single diastereomer: onlythe transcyclopropane was formed and the reduction step proceeded with total stereoselectivity (Scheme 1).

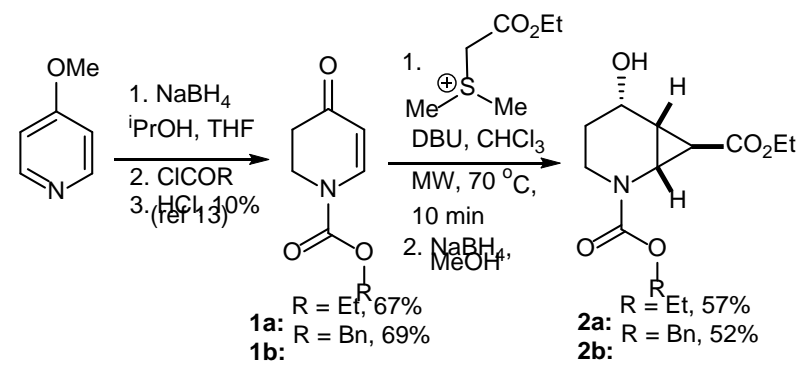

Scheme 1. Synthesis of cyclopropanes 2

The next step was the dehydration of compounds $\mathbf{2 a - b}$, which was accomplished by seleniation and oxidation-elimination to give 3a-b in good yields (78-83 \%). From these intermediates, an osmylation reaction gave two different syn-dihydroxy diastereomers from each substrate, which could be separated and characterized using 2D standard NMR experiments. At this point we had 4 compounds that were finally reduced using $\mathrm{LiBH}_{4}$ to give compounds 5 (Scheme 2). The final reduction of the ester moiety gave novel trihydroxylated bicyclic iminosugars bearing 5 stereogenic centers, which will be sent for evaluation against glycosidases. Figure 1 shows a 3D model of $\mathbf{6 a}$ where the flattened conformation is shown.

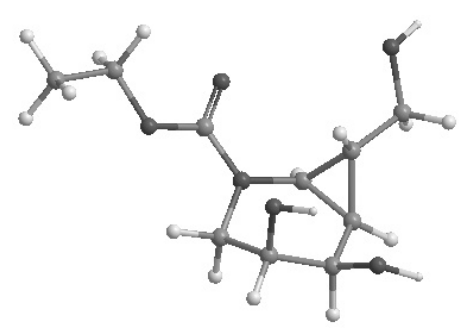

Figure 1. 3D model of 6 a

In conclusion, we show the synthesis of novel rigidified iminosugars bearing a cyclopropane ring, a carbamate substituent and 3 hydroxy groups as potential inhibitors of glycosidases. The method described will be used to obtain other highly functionalized frameworks containing the cyclopropane ring as intermediates 3-5 can readily be further transformed in other derivatives.

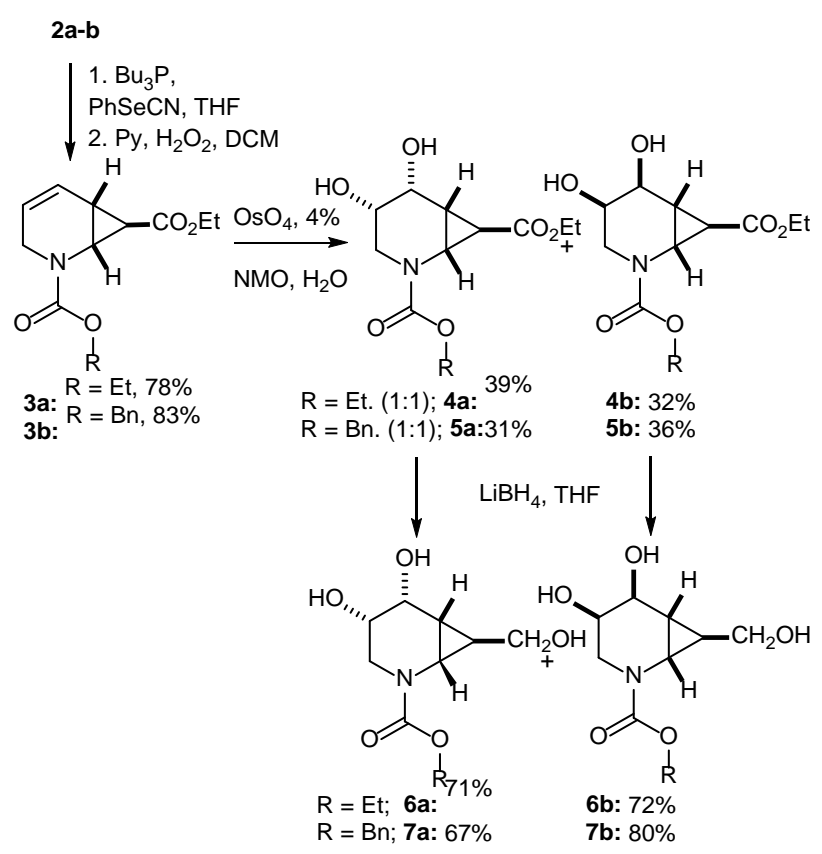

Scheme 2. Synthesis of compounds 6 and 7

\section{The experimental section has no title; please leave this line here.}

General: Reaction progress was monitored using analytical thinlayer chromatography (TLC) on Merck silica gel 60 F-254 plate. Visualization was achieved by UV light ( $254 \mathrm{~nm}$ ). Cyclopropanation reactions were carried out in a Biotage Initiator+ microwave reactor. NMR spectra were recorded on a Bruker spectrometer (400 $\mathrm{MHz}$ for ${ }^{1} \mathrm{H}$, and $101 \mathrm{MHz}$ for ${ }^{13} \mathrm{C}$ ). Chemical shifts are reported in $\delta$ ppm referenced to chloroform- $d\left(\delta 7.26\right.$ for ${ }^{1} \mathrm{H}$ NMR and $\delta 77.00$ for ${ }^{13} \mathrm{C}$ NMR) or Methanol- $d 4$ ( $\delta 3.31$ for ${ }^{1} \mathrm{H}$ NMR and $\delta$ 49.00 for ${ }^{13} \mathrm{C} \mathrm{NMR}$ ). All the residues were purified by flash chromatography on silica gel. Bidimensional spectra (HMQC, HMBC, COSY, NOESY) were recorded for all compounds in order to carry out the assignment.

\section{Procedures}


$\left(1 R^{*}, 5 S^{*}, 6 R^{*}, 7 R^{*}\right)$-Diethyl 5-hydroxy-2-azabicyclo[4.1.0]heptane2,7-dicarboxylate, 2a: From $4 \mathrm{~mL}(40.0 \mathrm{mmol})$ of 4-methoxypyridine, $4.53 \mathrm{~g}$ (26.8 mmol, 67\%) of ethyl 4-oxo-3,4-dihydropyridine-1(2H)carboxylate 1a were obtained following the procedure described in the literature except that $\mathrm{NaBH}_{4}$ was used as the reducing agent. ${ }^{14}$ To a solution of $10.22 \mathrm{~g} \quad(44.6 \mathrm{mmol})$ of (2-ethoxy-2oxoethyl)dimethylsulfonium bromide in $41 \mathrm{~mL}$ of $\mathrm{CHCl}_{3}, 6.75 \mathrm{~mL}$ (45.18 mmol) of DBU were added. After 30 minutes of stirring, $4.53 \mathrm{~g}$ of $1 \mathrm{a}$ were added and the reaction mixture was heated up to $70^{\circ} \mathrm{C}$ through microwave irradiation during $10 \mathrm{~min}$. The mixture was then washed with $\mathrm{HCl} 1 \mathrm{M}(30 \mathrm{~mL})$, water $(2 \times 30 \mathrm{~mL})$ and brine $(2 \times 30 \mathrm{~mL})$, dried with $\mathrm{MgSO}_{4}$, filtered and concentrated under vacuum to obtain $6.56 \mathrm{~g}$ of a crude mixture containing $\left(1 R^{*}, 6 R^{*}, 7 R^{*}\right)$-diethyl 5-oxo-2azabicyclo[4.1.0]heptane-2,7-dicarboxylate. This mixture was dissolved in $70 \mathrm{~mL}$ of methanol and $985 \mathrm{mg}(26.1 \mathrm{mmol})$ of $\mathrm{NaBH}_{4}$ were added in portions at $0^{\circ} \mathrm{C}$. After 45 minutes of stirring the reaction was quenched with $86 \mathrm{~mL}$ of water. The mixture was then extracted with diethyl ether (3x60 mL) and the organic phases were washed with brine $(60 \mathrm{~mL})$ dried with $\mathrm{MgSO}_{4}$, filtered and concentrated. After flash column chromatography (Hexane:EtOAc (2:1) $\rightarrow$ Hexane:EtOAc (1:2)) $3.95 \mathrm{~g}$ (15.2 mmol, $57 \%$ of yield from $1 \mathrm{a}, \mathrm{R}_{\mathrm{f}}=0.2$ in Hexane/EtOAc, $1: 1$ ) of a pure compound were obtained as a colorless oil. The compound was assigned as $\left(1 R^{*}, 5 S^{*}, 6 R^{*}, 7 R^{*}\right)$ due to the detection of NOESY cross peaks between $\mathrm{H} 5$ andH6. Spectra showed a mixture of two conformers $(67 \%$ $(\alpha): 33 \%(\beta))$

\section{IR: $3442,3017,1716,1689 \mathrm{~cm}^{-1}$.}

${ }^{1} \mathrm{H}$ NMR (400 MHz, $\left.\mathrm{CDCl}_{3}\right) \delta=4.32-4.24(\mathrm{~m}, 1 \mathrm{H}, \mathrm{H} 5), 4.20-4.03(\mathrm{~m}$, $\left.4 \mathrm{H}, \mathrm{CH}_{2} \mathrm{CH}_{3}\right), 3.85(\mathrm{dt}, J=13.1,3.1 \mathrm{~Hz}, 1 \mathrm{H}, \mathrm{H} 3$, conformer $\alpha), 3.71(\mathrm{dt}, J=$ 13.4, $3.8 \mathrm{~Hz}, 1 \mathrm{H}, \mathrm{H} 3$, conformer $\beta$ ), $3.54(\mathrm{dd}, J=8.8,2.7 \mathrm{~Hz}, 1 \mathrm{H}, \mathrm{H} 1$, conformer $\beta$ ), 3.41 (dd, $J=8.8,2.7 \mathrm{~Hz}, 1 \mathrm{H}, \mathrm{H} 1$, conformer $\alpha$ ), $2.80(\mathrm{~m}, 2 \mathrm{H}$, $\mathrm{OH}+\mathrm{H} 3$ conformer $\beta), 2.71(\mathrm{t}, J=12.7 \mathrm{~Hz}, 1 \mathrm{H}, \mathrm{H} 3$, conformer $\alpha), 2.19-$ $2.12(\mathrm{~m}, 1 \mathrm{H}, \mathrm{H} 6), 2.03-1.96(\mathrm{~m}, 1 \mathrm{H}, \mathrm{H} 4$ conformer $\beta), 1.94-1.85(\mathrm{~m}, 1 \mathrm{H}$, $\mathrm{H} 4$, conformer $\alpha), 1.85-1.78(\mathrm{~m}, 1 \mathrm{H}, \mathrm{H} 7), 1.30-1.15\left(\mathrm{~m}, 7 \mathrm{H}, \mathrm{CO}_{2} \mathrm{CH}_{2} \mathrm{CH}_{3}\right.$ $+\mathrm{NCO}_{2} \mathrm{CH}_{2} \mathrm{CH}_{3}+\mathrm{H} 4$ )

${ }^{13} \mathrm{C}$ NMR $\left(101 \mathrm{MHz}, \mathrm{CDCl}_{3}\right) \delta=171.5\left(\mathrm{CO}_{2}\right.$, conformer $\left.\alpha\right), 171.4\left(\mathrm{CO}_{2}\right.$, conformer $\beta), 156.5\left(\mathrm{NCO}_{2}\right.$, conformer $\left.\alpha\right), 156.1\left(\mathrm{NCO}_{2}\right.$, conformer $\left.\beta\right)$, 63.7 (C5, conformer $\alpha$ ), 63.5 ( $\mathrm{C} 5$, conformer $\beta$ ), $61.7\left(\mathrm{CH}_{2} \mathrm{CH}_{3}\right.$, conformer $\alpha), 61.6\left(\mathrm{CH}_{2} \mathrm{CH}_{3}\right.$, conformer $\left.\beta\right), 60.8\left(\mathrm{CH}_{2} \mathrm{CH}_{3}\right.$, conformer $\left.\beta\right), 60.7$ $\left(\mathrm{CH}_{2} \mathrm{CH}_{3}\right.$, conformer $\left.\alpha\right), 41.0$ (C3, conformer $\beta$ ), 40.7 (C3, conformer $\alpha$ ), 40.6 (C1, conformer $\beta$ ), 40.2 (C1, conformer $\alpha$ ), 30.7 (C4, conformer $\alpha$ ), 30.5 (C4, conformer $\beta$ ), 27.8 (C6, conformer $\alpha$ ), 27.7 (C6, conformer $\beta$ ), 25.2 (C7, conformer $\alpha$ ), 24.5 (C7, conformer $\beta$ ), $14.4\left(\mathrm{CH}_{2} \mathrm{CH}_{3}\right), 14.2$ $\left(\mathrm{CH}_{2} \mathrm{CH}_{3}\right.$, conformer $\left.\alpha\right), 14.0\left(\mathrm{CH}_{2} \mathrm{CH}_{3}\right.$, conformer $\left.\beta\right)$.

Anal. Calcd. for $\mathrm{C}_{12} \mathrm{H}_{19} \mathrm{NO}_{5}(257.28 \mathrm{~g} / \mathrm{mol}): \mathrm{C}, 56.0 ; \mathrm{H}, 7.4 \%$. Found: $\mathrm{C}$ $56.2 ; \mathrm{H}, 7.0 \%$.

\section{$\left(1 R^{*}, 5 S^{*}, 6 R^{*}, 7 R^{*}\right)$-2-Benzyl 7-ethyl 5-hydroxy-2-} azabicyclo[4.1.0]heptane-2,7-dicarboxylate, 2b: From $4.0 \mathrm{~mL}(40.0$ mmol) of 4-methoxy pyridine, $6.38 \mathrm{~g}(27.6 \mathrm{mmol}, 69 \%)$ of benzyl 4-oxo3,4-dihydropyridine-1 $(2 \mathrm{H})$-carboxylate $\mathbf{1 b}$ were obtained, following the procedure described in the literature except that $\mathrm{NaBH}_{4}$ was used as the reducing agent. ${ }^{14}$ The synthesis was continued as for compound 2a using $10.0 \mathrm{~g}$ (43.75 mmol) of (2-ethoxy-2-oxoethyl)dimethylsulfonium bromide in $45 \mathrm{~mL}$ of $\mathrm{CHCl}_{3}$ and $7 \mathrm{~mL}(46.00 \mathrm{mmol})$ of DBU. After the cyclopropanation step, $7.0 \mathrm{~g}$ of crude $\left(1 R^{*}, 6 R^{*}, 7 R^{*}\right)$-2-benzyl 7-ethyl 5oxo-2-azabicyclo[4.1.0]heptane-2,7-dicarboxylate were treated in $70 \mathrm{~mL}$ of methanol with $1.024 \mathrm{~g}(27.1 \mathrm{mmol})$ of $\mathrm{NaBH}_{4}$ giving, after isolation and flash column chromatography (Hexane:EtOAc (2:1) $\rightarrow$ Hexane:EtOAc (1:2)) $3.67 \mathrm{~g}$ (14.35 mmol, $52 \%$ yield from $\mathbf{1 b}, \mathrm{Rf}=0.1$ in Hexane/EtOAc, $1: 1)$ of $\mathbf{2 b}$ as a colorless oil. This compound was assigned as $\left(1 R^{*}, 5 S^{*}, 6 R^{*}, 7 R^{*}\right)$ due to the detection of NOESY cross peaks between $\mathrm{H} 5$ and $\mathrm{H} 6$ and analogy with compound 2a. Spectra showed a mixture of two conformers $(66 \%(\alpha): 34 \%(\beta))$.

IR: 3460, 3022, 2959, $1699 \mathrm{~cm}^{-1}$.

${ }^{1} \mathrm{H}$ NMR (400 MHz, $\left.\mathrm{CDCl}_{3}\right) \delta 7.43-7.27(\mathrm{~m}, 5 \mathrm{H}, \mathrm{CH} \mathrm{Ar}), 5.28-5.04(\mathrm{~m}$, $2 \mathrm{H}, \mathrm{CH}_{2} \mathrm{Ar}$ ), $4.39-4.28(\mathrm{~m}, 1 \mathrm{H}, \mathrm{H} 5), 4.18-4.02\left(\mathrm{~m}, 2 \mathrm{H}, \mathrm{CO}_{2} \mathrm{CH}_{2} \mathrm{CH}_{3}\right), 3.94$ $(\mathrm{dt}, J=13.5,3.7 \mathrm{~Hz}, 1 \mathrm{H}, \mathrm{H} 3$, conformer $\alpha), 3.83-3.75(\mathrm{~m}, 1 \mathrm{H}, \mathrm{H} 3$, conformer $\beta$ ), $3.63(\mathrm{~d}, J=9.4 \mathrm{~Hz}, 1 \mathrm{H}, \mathrm{H} 1$, conformer $\beta), 3.54(\mathrm{~d}, J=8.8 \mathrm{~Hz}$,
$1 \mathrm{H}, \mathrm{H} 1$, conformer $\alpha), 2.87(\mathrm{t}, J=13.3 \mathrm{~Hz}, 1 \mathrm{H}, \mathrm{H} 3$, conformer $\beta), 2.78(\mathrm{t}, J$ $=13.2 \mathrm{~Hz}, 1 \mathrm{H}, \mathrm{H} 3$, conformer $\alpha), 2.20(\mathrm{~m}, 1 \mathrm{H}, \mathrm{H} 6), 2.05-1.80(\mathrm{~m}, 1 \mathrm{H}$, H4), 1.87 (dd, $J=5.9,2.7 \mathrm{~Hz}, 1 \mathrm{H}, \mathrm{H} 7), 1.30-1.21\left(\mathrm{~m}, 4 \mathrm{H}, \mathrm{CH}_{2} \mathrm{CH}_{3}\right.$ conformer $\beta+\mathrm{H} 4), 1.20\left(\mathrm{t}, J=7.0 \mathrm{~Hz}, 3 \mathrm{H}, \mathrm{CH}_{2} \mathrm{CH}_{3}\right.$, conformer $\left.\alpha\right)$.

${ }^{13} \mathrm{C}$ NMR (101 MHz, $\left.\mathrm{CDCl}_{3}\right) \delta 171.3\left(\mathrm{CO}_{2} \mathrm{Et}\right), 156.2\left(\mathrm{NCO}_{2}\right), 136.4(\mathrm{C} \mathrm{Ar}$, conformer $\alpha$ ) 135.9 (C Ar, conformer $\beta$ ), 128.5 ( $\mathrm{CH} \mathrm{Ar,} \mathrm{conformer} \alpha$ ), $128.3(\mathrm{CH} \mathrm{Ar}$, conformer $\alpha$ ), 128.2 ( $\mathrm{CH} \mathrm{Ar}$, conformer $\beta$ ), $128.0(\mathrm{CH} \mathrm{Ar}$, conformer $\beta$ ), 127.8 ( $\mathrm{CH} \mathrm{Ar}$, conformer $\beta$ ), 127.3 ( $\mathrm{CH} \mathrm{Ar}$, conformer $\alpha$ ), $67.8\left(\mathrm{CH}_{2}-\mathrm{Ar}\right.$, conformer $\left.\beta\right), 67.3\left(\mathrm{CH}_{2}-\mathrm{Ar}\right.$, conformer $\left.\alpha\right), 63.7(\mathrm{C} 5$, conformer $\alpha$ ), 63.5 (C5, conformer $\beta), 61.2\left(\mathrm{CO}_{2} \mathrm{CH}_{2}\right.$, conformer $\left.\beta\right), 60.8$ $\left(\mathrm{CO}_{2} \mathrm{CH}_{2}\right.$, conformer $\left.\alpha\right), 41.1$ (C3, conformer $\left.\beta\right), 40.9(\mathrm{C} 3$, conformer $\alpha)$, 40.7 (C1, conformer $\beta$ ), 40.2 (C1, conformer $\alpha$ ), 30.8 (C4, conformer $\alpha$ ), 30.4 (C4, conformer $\beta$ ), 27.8 (C6, conformer $\alpha$ ), 27.6 (C6, conformer $\beta$ ), 25.3 (C7, conformer $\alpha$ ), 24.4 ( $\mathrm{C} 7$, conformer $\beta$ ), $14.1\left(\mathrm{CH}_{2} \mathrm{CH}_{3}\right.$, conformer $\beta), 14.1\left(\mathrm{CH}_{2} \mathrm{CH}_{3}\right.$, conformer $\left.\alpha\right)$.

Anal. Calcd. for $\mathrm{C}_{17} \mathrm{H}_{21} \mathrm{NO}_{5}$ (319.35 g/mol): C, 63.9; H, 6.6 \%. Found: C, 64.1; H, $6.3 \%$.

$\left(1 R^{*}, 6 R^{*}, 7 R^{*}\right)$-Diethyl

2-azabicyclo[4.1.0]hept-4-ene-2,7dicarboxylate, 3a: To a solution of $1.20 \mathrm{~g}$ ( $4.7 \mathrm{mmol})$ of $2 \mathbf{a}$ in $30 \mathrm{~mL}$ of anhydrous THF, $2.40 \mathrm{~mL}$ ( $9.4 \mathrm{mmol})$ of tributylphosphine under Argon atmosphere were added. The solution was heated up to reflux temperature and another solution of phenyl selenocyanate $(1.25 \mathrm{~mL}, 9.4$ $\mathrm{mmol}$ in $8.20 \mathrm{~mL}$ of THF anh.) were then added to the system. The reaction mixture was then stirred overnight and concentrated under vacuum to give $1.30 \mathrm{~g}$ of a crude mixture. This crude was dissolved in 17 $\mathrm{mL}$ of DCM and $0.55 \mathrm{~mL}$ of pyridine followed by $783 \mu \mathrm{L}$ of $\mathrm{H}_{2} \mathrm{O}_{2} 33 \%$ were added and the mixture was stirred for 10 minutes. After this time, 3 $\mathrm{mL}$ of THF were added and the mixture was stirred for another 30 minutes. Then, $44 \mathrm{~mL}$ of diethyl ether were added and the mixture was washed once with $20 \mathrm{~mL}$ of a $10 \%$ solution of $\mathrm{Na}_{2} \mathrm{~S}_{2} \mathrm{O}_{4}$ in water, then with water $(20 \mathrm{~mL})$, the organic phase was dried over $\mathrm{MgSO}_{4}$, filtered and concentrated under vacuum. After flash column chromatography (Hexane:EtOAc, 9:1) $860 \mathrm{mg}$ (3.60 mmol, $78 \%$ of yield, $\mathrm{Rf}=0.2$ in Hexane/EtOAc, 9:1) of 3a were obtained as a yellow oil. Spectra showed a mixture of two conformers $(60 \%(\alpha): 40 \%(\beta))$.

IR: $3022,2987,1719 \mathrm{~cm}^{-1}$.

${ }^{1} \mathrm{H} \mathrm{NMR}\left(400 \mathrm{MHz}, \mathrm{CDCl}_{3}\right) \delta=6.08-5.98(\mathrm{~m}, 1 \mathrm{H}, \mathrm{H} 5), 5.80-5.72(\mathrm{~m}, 1 \mathrm{H}$, $\mathrm{H} 4$, conformer $\alpha), 5.71-5-63(\mathrm{~m}, 1 \mathrm{H}, \mathrm{H} 4$, conformer $\beta), 4.27-4.09(\mathrm{~m}$, $4 \mathrm{H}, \mathrm{CH}_{2} \mathrm{CH}_{3}$ ), 3.99 (dd, $J=18.3,5.0 \mathrm{~Hz}, 1 \mathrm{H}, \mathrm{H} 3$, conformer $\alpha$ ), $3.90-3.60$ (m, 4H, 2xH3 conformer $\beta+\mathrm{H} 3$ conformer $\alpha+\mathrm{H} 1$ conformer $\beta$ ), 3.53 (dd, $J=8.3,2.7 \mathrm{~Hz}, 1 \mathrm{H}, \mathrm{H} 1$, conformer $\alpha), 2.18-2.11(\mathrm{~m}, 1 \mathrm{H}, \mathrm{H} 6), 1.65-1.55$ (m, 1H, H7), 1.27 (t, $J=7.0 \mathrm{~Hz}, 6 \mathrm{H}, \mathrm{CH}_{2} \mathrm{CH}_{3}$ ).

${ }^{13} \mathrm{C}$ NMR $\left(101 \mathrm{MHz}, \mathrm{CDCl}_{3}\right) \delta=170.7\left(\mathrm{CO}_{2}\right), 156.6\left(\mathrm{NCO}_{2}\right.$, conformer $\left.\alpha\right)$ $156.4\left(\mathrm{NCO}_{2}\right.$, conformer $\beta$ ), $125.2(\mathrm{C} 4$, conformer $\alpha$ ), 123.9 (C4, conformer $\beta$ ), 122.4 (C5, conformer $\beta$ ), 122.1 (C5, conformer $\alpha$ ), 61.7 $\left(\mathrm{CH}_{2} \mathrm{CH}_{3}\right), 60.6\left(\mathrm{CH}_{2} \mathrm{CH}_{3}\right), 40.6(\mathrm{C} 3$, conformer $\beta), 40.1(\mathrm{C} 3$, conformer $\alpha)$, 36.7 ( $\mathrm{C} 1$, conformer $\beta), 36.40(\mathrm{C} 1$, conformer $\alpha), 34.7$ (C7, conformer $\alpha$ ), $34.2(\mathrm{C} 7$, conformer $\beta$ ), 21.5(C6, conformer $\alpha$ ), 20.6 (C6, conformer $\beta$ ), $14.6\left(\mathrm{CH}_{2} \mathrm{CH}_{3}\right), 14.3\left(\mathrm{CH}_{2} \mathrm{CH}_{3}\right)$.

Anal. Calcd. for $\mathrm{C}_{12} \mathrm{H}_{17} \mathrm{NO}_{4}(239.27 \mathrm{~g} / \mathrm{mol}): \mathrm{C}, 60.2 ; \mathrm{H}, 7.2 \%$. Found: C, $60.1 ; \mathrm{H}, 7.5 \%$.

$\left(1 R^{*}, 6 R^{*}, 7 R^{*}\right)$-2-Benzyl 7-ethyl 2-azabicyclo[4.1.0]hept-4-ene-2,7dicarboxylate, $\mathbf{3 b}$ : Following the same procedure as for $\mathbf{3 a}$, from $2.16 \mathrm{~g}$ (6.81 mmol) of $\mathbf{2 b}, 3.02 \mathrm{~mL}$ (12.2 mmol) of tributylphosphine, $1.67 \mathrm{~mL}$ (13.7 mmol) of phenyl selenocyanate, and after flash column chromatography (Hexane:EtOAc, 9:1), 1.70 g (5.65 mmol, 83 \% yield, Rf $=0.2$ in Hexane/EtOAc, 9:1) of $\mathbf{3 b}$ were obtained as a yellow oil. Spectra showed a mixture of two conformers $(65 \%(\alpha): 35 \%(\beta))$.

IR: $2983,1711,1699 \mathrm{~cm}^{-1}$.

${ }^{1} \mathrm{H}$ NMR (400 MHz, $\left.\mathrm{CDCl}_{3}\right) \delta=7.42-7.28(\mathrm{~m}, 5 \mathrm{H}, \mathrm{CH} \mathrm{Ar}), 6.07-5.95(\mathrm{~m}$, 1H, H5), $5.83-5.73(\mathrm{~m}, 1 \mathrm{H}, \mathrm{H} 4$, conformer $\alpha), 5.69-5.63(\mathrm{~m}, 1 \mathrm{H}, \mathrm{H} 4$, conformer $\beta$ ), 5.27 - $5.12\left(\mathrm{~m}, 2 \mathrm{H}, \mathrm{CH}_{2}-\mathrm{Ar}\right), 4.19-4.04\left(\mathrm{~m}, 2 \mathrm{H}, \mathrm{CH}_{2} \mathrm{CH}_{3}\right)$, $4.02(\mathrm{dd}, J=18.5,5.5 \mathrm{~Hz}, 1 \mathrm{H}, \mathrm{H} 3$, conformer $\alpha), 3.95-3.75(\mathrm{~m}, 2 \mathrm{H}, \mathrm{H} 3$, conformer $\beta$ ), $3.73(\mathrm{dd}, J=8.7,2.1 \mathrm{~Hz}, 1 \mathrm{H}, \mathrm{H} 1$, conformer $\beta$ ), $3.71-3.61$ (m, $1 \mathrm{H}, \mathrm{H} 3$, conformer $\alpha$ ), $3.58(\mathrm{dd}, J=8.1,2.7 \mathrm{~Hz}, 1 \mathrm{H}, \mathrm{H} 1$, conformer $\alpha$ ), $2.22-2.13(\mathrm{~m}, 1 \mathrm{H}, \mathrm{H} 6), 1.67-1.64(\mathrm{~m}, 1 \mathrm{H}, \mathrm{H} 7$, conformer $\beta), 1.62(\mathrm{dd}, J$ 
= 5.0, $2.7 \mathrm{~Hz}, 1 \mathrm{H}, \mathrm{H} 7$, conformer $\alpha$ ), $1.26\left(\mathrm{t}, J=7.1 \mathrm{~Hz}, 3 \mathrm{H}, \mathrm{CH}_{2} \mathrm{CH}_{3}\right.$, conformer $\beta$ ), $1.21\left(\mathrm{t}, J=7.1 \mathrm{~Hz}, 3 \mathrm{H}, \mathrm{CH}_{2} \mathrm{CH}_{3}\right.$, conformer $\alpha$ ).

${ }^{13} \mathrm{C}$ NMR $\left(101 \mathrm{MHz}, \mathrm{CDCl}_{3}\right) \delta=170.5\left(\mathrm{CO}_{2}\right.$, conformer $\left.\alpha\right), 170.4\left(\mathrm{CO}_{2}\right.$, conformer $\beta), 156.3\left(\mathrm{NCO}_{2}\right.$, conformer $\left.\alpha\right), 156.1\left(\mathrm{NCO}_{2}\right.$, conformer $\left.\beta\right)$, 136.5(C Ar, conformer $\alpha$ ), 136.3 (C Ar, conformer $\beta$ ) $128.6(\mathrm{CH} \mathrm{Ar}$, conformer $\alpha$ ), 128.4 ( $\mathrm{CH} \mathrm{Ar}$, conformer $\beta$ ) $128.4(\mathrm{CH} \mathrm{Ar}$, conformer $\alpha$ ), $128.2(\mathrm{CH} \mathrm{Ar}$, conformer $\beta$ ), $127.9(\mathrm{CH} \mathrm{Ar}$, conformer $\beta$ ), $127.4(\mathrm{CH} \mathrm{Ar}$, conformer $\alpha$ ), 125.1 (C4, conformer $\alpha$ ), 123.7 (C4, conformer $\beta$ ), 122.4 (C5, conformer $\beta$ ), 122.0 ( $\mathrm{C} 5$, conformer $\alpha$ ), $67.4\left(\mathrm{CH}_{2}-\mathrm{Ar}\right.$, conformer $\beta$ ), $67.2\left(\mathrm{CH}_{2}-\mathrm{Ar}\right.$, conformer $\left.\alpha\right), 60.7\left(\mathrm{CH}_{2} \mathrm{CH}_{3}\right), 40.7(\mathrm{C} 3$, conformer $\beta), 40.2$ (C3, conformer $\alpha$ ), 36.8 (C1, conformer $\beta$ ), 36.3 (C1, conformer $\alpha$ ), 34.8 (C7, conformer $\alpha$ ), 34.2 (C7, conformer $\beta$ ), 21.5 (C6, conformer $\alpha$ ), 20.6 (C6, conformer $\beta), 14.2\left(\mathrm{CH}_{2} \mathrm{CH}_{3}\right.$, conformer $\left.\alpha\right), 14.1\left(\mathrm{CH}_{2} \mathrm{CH}_{3}\right.$, conformer $\beta)$.

Anal. Calcd. for $\mathrm{C}_{17} \mathrm{H}_{19} \mathrm{NO}_{4}(301.34 \mathrm{~g} / \mathrm{mol})$ : C, 67.8; H, $6.4 \%$. Found: C, 68.0; H, $6.7 \%$.

Diethyl 4,5-dihydroxy-2-azabicyclo[4.1.0]heptane-2,7dicarboxylate: To a solution of $430 \mathrm{mg}(1.80 \mathrm{mmol})$ of $3 \mathbf{a}$ in $7.30 \mathrm{~mL}$ of acetone were added, sequentially, $2.23 \mathrm{~mL}(0.36 \mathrm{mmol})$ of $\mathrm{OsO}_{4} 4 \%$ in water and a solution of 4-methylmorpholine $N$-oxide $(316.30 \mathrm{mg}$ $2.70 \mathrm{mmol}$ ) in $316.30 \mu \mathrm{L}$ of water. The resulting mixture was stirred overnight at r.t. Then, one spatula of $\mathrm{Na}_{2} \mathrm{SO}_{3}$ and another of $\mathrm{Na}_{2} \mathrm{SO}_{4}$ were added and the acetone was evaporated in vacuum. The mixture was then extracted with EtOAc $(3 \times 3 \mathrm{~mL})$, washed with brine $(5 \mathrm{~mL})$, the organic phase was dried over $\mathrm{MgSO}_{4}$, filtered and evaporated to give a crude (1:1) mixture of two diastereoisomers. After flash column chromatography (Hexane:EtOAc (1:2)) both isomers were separated and characterized.

$\left(\mathbf{1} \boldsymbol{R}^{*}, \mathbf{4} \boldsymbol{S}^{*}, \mathbf{5} \boldsymbol{R}^{*}, \mathbf{6} \boldsymbol{R}^{*}, \mathbf{7} \boldsymbol{R}^{*}\right)$-isomer, $\mathbf{4 a} 183 \mathrm{mg}$ (0.68 mmol, $39 \%$ of yield, $\mathrm{Rf}=0.26$ in Hexane/EtOAc, 1:2) were obtained as a colorless oil. The compound was assigned as $\left(1 R^{*}, 4 S^{*}, 5 R^{*}, 6 R^{*}, 7 R^{*}\right)$ due to strong NOESY cross peaks between $\mathrm{H} 5$ and H6. Spectra showed a mixture of two conformers $(70 \%(\alpha): 30 \%(\beta))$.

IR: $3429,2983,2920,1707 \mathrm{~cm}^{-1}$

${ }^{1} \mathrm{H}$ NMR (400 MHz, $\mathrm{CDCl}_{3}$ ) $\delta=4.32-4.28(\mathrm{~m}, 1 \mathrm{H}, \mathrm{H} 5), 4.23-4.09(\mathrm{~m}, 4 \mathrm{H}$, $\left.\mathrm{CH}_{2} \mathrm{CH}_{3}\right), 3.74(\mathrm{dd}, J=12.1,4.1 \mathrm{~Hz}, 1 \mathrm{H}, \mathrm{H} 3$, conformer $\alpha$ ), $3.61(\mathrm{dd}, J=$ 11.7, $2.2 \mathrm{~Hz}, 1 \mathrm{H}, \mathrm{H} 3$, conformer $\beta), 3.55-3.45(\mathrm{~m}, 2 \mathrm{H}, \mathrm{H} 4+\mathrm{H} 1$ conformer $\beta$ ), $3.38(\mathrm{dd}, J=8.2,2.0 \mathrm{~Hz}, 1 \mathrm{H}, \mathrm{H} 1$, conformer $\alpha), 3.05(\mathrm{t}, J=$ $11.7 \mathrm{~Hz}, 1 \mathrm{H}, \mathrm{H} 3$, conformer $\beta$ ), $2.99(\mathrm{t}, J=12.1 \mathrm{~Hz}, 1 \mathrm{H}, \mathrm{H} 3$, conformer $\alpha$ ), 2.60 (brs, 1H, OH), 2.19 (t, J=7.2 Hz, 1H, H6), 1.58 (br s, 1H, OH), 1.51 $1.38(\mathrm{~m}, 1 \mathrm{H}, \mathrm{H} 7), 1.27\left(\mathrm{t}, J=7.0 \mathrm{~Hz}, 3 \mathrm{H}, \mathrm{CH}_{2} \mathrm{CH}_{3}\right), 1.25(\mathrm{t}, J=7.0 \mathrm{~Hz}, 3 \mathrm{H}$, $\left.\mathrm{CH}_{2} \mathrm{CH}_{3}\right)$.

${ }^{13} \mathrm{C}$ NMR (101 MHz, $\mathrm{CDCl}_{3}$, data of major conformer) $\delta=171.0\left(\mathrm{CO}_{2}\right)$, $156.6\left(\mathrm{NCO}_{2}\right), 67.2$ (C4), 64.5 (C5), $62.0\left(\mathrm{CH}_{2} \mathrm{CH}_{3}\right), 60.9\left(\mathrm{CH}_{2} \mathrm{CH}_{3}\right), 41.0$ (C3), 36.9 (C1), 27.9 (C6), 26.0 (C7), $14.5\left(\mathrm{CH}_{2} \mathrm{CH}_{3}\right), 14.3\left(\mathrm{CH}_{2} \mathrm{CH}_{3}\right)$.

Anal. Calcd. for $\mathrm{C}_{12} \mathrm{H}_{19} \mathrm{NO}_{6}(273.28 \mathrm{~g} / \mathrm{mol}): \mathrm{C}, 52.7 ; \mathrm{H}, 7.0 \%$. Found: $\mathrm{C}$, $53.1 ; \mathrm{H}, 7.3 \%$.

$\left(1 \boldsymbol{R}^{*}, \mathbf{4} \boldsymbol{R}^{*}, \mathbf{5} \boldsymbol{S}^{*}, \mathbf{6} \boldsymbol{R}^{*}, \mathbf{7} \boldsymbol{R}^{*}\right)$-isomer, $\mathbf{4 b} \mathbf{b} 151 \mathrm{mg}(0.56 \mathrm{mmol}, 32 \%$ of yield $\mathrm{Rf}=0.18$ in Hexane/EtOAc, 1:2) were obtained as a colorless oil. The compound was assigned as $\left(1 R^{*}, 4 R^{*}, 5 S^{*}, 6 R^{*}, 7 R^{*}\right)$ due to the absence of NOESY cross peaks between H5 and H6. Spectra showed a mixture of two conformers $(80 \%(\alpha): 20 \%(\beta))$.

IR: 3431, 2985, 2921, $1704 \mathrm{~cm}^{-1}$

${ }^{1} \mathrm{H}$ NMR (400 MHz, $\left.\mathrm{CDCl}_{3}\right) \delta=4.27-4.08\left(\mathrm{~m}, 6 \mathrm{H}, 2 \mathrm{xCH}_{2} \mathrm{CH}_{3}+\mathrm{H} 3+\mathrm{H} 5\right)$, $4.03(\mathrm{t}, J=4.0 \mathrm{~Hz}, 1 \mathrm{H}, \mathrm{H} 4$, conformer $\alpha$ ), 3.97 (br s, $1 \mathrm{H}, \mathrm{H} 4$, conformer $\beta$ ), $3.52(\mathrm{~d}, J=9.3 \mathrm{~Hz}, 1 \mathrm{H}, \mathrm{H} 1$, conformer $\beta$ ), 3.42 (dd, $J=8.5,2.6 \mathrm{~Hz}, 1 \mathrm{H}, \mathrm{H} 1$, conformer $\alpha$ ), $2.97(\mathrm{~d}, J=13.1 \mathrm{~Hz}, 1 \mathrm{H}, \mathrm{H} 3$, conformer $\beta$ ), $2.86(\mathrm{~d}, J=14.0$ $\mathrm{Hz}, 1 \mathrm{H}, \mathrm{H} 3$, conformer $\alpha$ ), 2.46 (br s, $1 \mathrm{H}, \mathrm{C} 5-\mathrm{OH}$ ), 2.37 (br s, 1H, C4-OH), 2.35 (dd, $J=5.8,2.6 \mathrm{~Hz}, 1 \mathrm{H}, \mathrm{H} 7), 2.13(\mathrm{~m}, 1 \mathrm{H}, \mathrm{H} 6), 1.27$ (t, $J=7.0 \mathrm{~Hz}, 3 \mathrm{H}$, $\left.\mathrm{CH}_{2} \mathrm{CH}_{3}\right), 1.26\left(\mathrm{t}, J=7.1 \mathrm{~Hz}, 3 \mathrm{H}, \mathrm{CH}_{2} \mathrm{CH}_{3}\right)$.

${ }^{13} \mathrm{C}$ NMR (101 MHz, $\mathrm{CDCl}_{3}$, data of major conformer) $\delta=171.7\left(\mathrm{CO}_{2}\right)$, $157.8\left(\mathrm{NCO}_{2}\right), 67.8$ (C4), 64.4 (C5), $61.9\left(\mathrm{CH}_{2} \mathrm{CH}_{3}\right), 60.7\left(\mathrm{CH}_{2} \mathrm{CH}_{3}\right), 46.2$ (C3), 39.9 (C1), 26.8 (C7), 26.6 (C6), $14.5\left(\mathrm{CH}_{2} \mathrm{CH}_{3}\right), 14.3\left(\mathrm{CH}_{2} \mathrm{CH}_{3}\right)$.

Anal. Calcd. for $\mathrm{C}_{12} \mathrm{H}_{19} \mathrm{NO}_{6}(273.28 \mathrm{~g} / \mathrm{mol}): \mathrm{C}, 52.7 ; \mathrm{H}, 7.0 \%$. Found: $\mathrm{C}$, 52.6; $\mathrm{H}, 6.8 \%$.
2-Benzyl 7-ethyl 4,5-dihydroxy-2-azabicyclo[4.1.0]heptane-2,7dicarboxylate: Following the same procedure as for 4, from $400 \mathrm{mg}$ ( $1.33 \mathrm{mmol})$ of $3 \mathbf{b}, 2.69 \mathrm{~mL}(0.26 \mathrm{mmol})$ of $\mathrm{OsO}_{4} 4 \%$ in water and 230.5 mg $(1.97 \mathrm{mmol})$ of 4 -methylmorpholine $N$-oxide in $230 \mu \mathrm{L}$ of water, a crude (1:1) mixture of two diastereoisomers was obtained which after flash column chromatography (Hexane:EtOAc (1:2)) were separated and characterized.

$\left(\mathbf{1} \boldsymbol{R}^{*}, \mathbf{4} \boldsymbol{S}^{*}, \mathbf{5} \boldsymbol{R}^{*}, \mathbf{6} \boldsymbol{R}^{*}, \mathbf{7} \boldsymbol{R}^{*}\right)$-isomer, 5a: $138 \mathrm{mg}(0.41 \mathrm{mmol}, 31 \%$ yield, $\mathrm{Rf}=0.35$ in Hexane/EtOAc, 1:2) as a yellow oil. The product was assigned as $\left(1 R^{*}, 4 S^{*}, 5 R^{*}, 6 R^{*}, 7 R^{*}\right)$ due to detection of NOESY cross peaks between $\mathrm{H} 5$ and $\mathrm{H} 6$ and analogy with compound $\mathbf{4 a}$. Spectra showed a mixture of two conformers $(80 \%(\alpha): 20 \%(\beta))$.

IR: $3427,2980,2924,1702 \mathrm{~cm}^{-1}$.

${ }^{1} \mathrm{H}$ NMR (400 MHz, $\left.\mathrm{CDCl}_{3}\right) \delta=7.39-7.28(\mathrm{~m}, 5 \mathrm{H}, \mathrm{CH} \mathrm{Ar}), 5.25-5.08(\mathrm{~m}$, $2 \mathrm{H}, \mathrm{CH}_{2} \mathrm{Ar}$ ), 4.29 (br s, $1 \mathrm{H}, \mathrm{H} 5$ ), $4.20-4.02$ (m, 2H, $\mathrm{CH}_{2} \mathrm{CH} 3$ ), 3.75 (dd, $J=$ 12.4, 4.1 Hz, $1 \mathrm{H}, \mathrm{H} 3$, conformer $\alpha), 3.63(\mathrm{dd}, J=12.4,4.0 \mathrm{~Hz}, 1 \mathrm{H}$, H3, conformer $\beta$ ), $3.55-3.46(\mathrm{~m}, 1 \mathrm{H}, \mathrm{H} 4), 3.44(\mathrm{dd}, J=8.2,2.5 \mathrm{~Hz}, 1 \mathrm{H}$, $\mathrm{H} 1), 3.07(\mathrm{t}, J=11.2 \mathrm{~Hz}, 1 \mathrm{H}, \mathrm{H} 3$, conformer $\beta$ ), $3.00(\mathrm{dd}, J=12.4,10.8 \mathrm{~Hz}$, $1 \mathrm{H}, \mathrm{H} 3$, conformer $\alpha$ ), $2.84-2.68(\mathrm{~m}, 1 \mathrm{H}, \mathrm{OH}), 2.22-2.15(\mathrm{~m}, 1 \mathrm{H}, \mathrm{H} 6)$, 1.46 (dd, $J=6.1,2.6 \mathrm{~Hz}, 1 \mathrm{H}, \mathrm{H} 7), 1.29-1.23\left(\mathrm{~m}, 3 \mathrm{H}, \mathrm{CH}_{2} \mathrm{CH}_{3}\right.$, conformer ß), $1.19\left(\mathrm{t}, J=7.1 \mathrm{~Hz}, 1 \mathrm{H}, \mathrm{CH}_{2} \mathrm{CH}_{3}\right.$, conformer $\alpha$ ).

${ }^{13} \mathrm{C}$ NMR $\left(101 \mathrm{MHz}, \mathrm{CDCl}_{3}\right) \delta=170.9\left(\mathrm{CO}_{2} \mathrm{Et}\right), 156.3\left(\mathrm{NCO}_{2}\right), 136.2(\mathrm{C} \mathrm{Ar}$, conformer $\alpha$ ), $136.0(C \mathrm{Ar}$, conformer $\beta$ ), $128.6(\mathrm{CH}$ Ar, conformer $\beta$ ), $128.5(\mathrm{CH} \mathrm{Ar}$, conformer $\alpha), 128.3(\mathrm{CH} \mathrm{Ar}$, conformer $\beta), 128.1(\mathrm{CH} \mathrm{Ar}$, conformer $\beta$ ), $128.0(\mathrm{CH} \mathrm{Ar}$, conformer $\alpha$ ), $127.5(\mathrm{CH} \mathrm{Ar}$, conformer $\alpha$ ), $67.7\left(\mathrm{CH}_{2}-\mathrm{Ar}\right.$, conformer $\left.\beta\right), 67.6\left(\mathrm{CH}_{2}-\mathrm{Ar}\right.$, conformer $\left.\alpha\right)$, 67.1 (C4, conformer $\alpha$ ), 67.0 ( $\mathrm{C} 4$, conformer $\beta$ ), 64.5 (C5), $61.1\left(\mathrm{CO}_{2} \mathrm{CH}_{2} \mathrm{CH}_{3}\right.$, conformer $\beta$ ), $61.0\left(\mathrm{CO}_{2} \mathrm{CH}_{2} \mathrm{CH}_{3}\right.$, conformer $\left.\alpha\right), 41.9$ (C3, conformer $\beta$ ), 41.1 (C3, conformer $\alpha$ ), 37.2 (C1, conformer $\beta$ ), 36.8 (C1, conformer $\alpha$ ), 27.9 (C6, conformer $\alpha$ ), 27.4 (C6, conformer $\beta$ ), 26.0 (C7, conformer $\alpha$ ), 25.3 (C7, conformer $\beta$ ), $14.1\left(\mathrm{CH}_{2} \mathrm{CH}_{3}\right)$.

Anal. Calcd. for $\mathrm{C}_{17} \mathrm{H}_{21} \mathrm{NO}_{6}$ (335.35 g/mol): C, 60.9; H, $6.3 \%$. Found: $\mathrm{C}$, $61.0 ; \mathrm{H}, 6.0 \%$.

$\left(\mathbf{1} \boldsymbol{S}^{*}, \mathbf{4} \boldsymbol{R}^{*}, \mathbf{5} \boldsymbol{S}^{*}, \mathbf{6} \boldsymbol{R}^{*}, \mathbf{7} \boldsymbol{R}^{*}\right)$-isomer, $\mathbf{5 b}: 156 \mathrm{mg}(0.47 \mathrm{mmol}, 36 \%$ yield, $\mathrm{Rf}=0.3$ in Hexane/EtOAc, $1: 3$ ) as a yellow oil. The product was assigned as $\left(1 S^{*}, 4 R^{*}, 5 S^{*}, 6 R^{*}, 7 R^{*}\right)$-due to absence of NOESY cross peaks between $\mathrm{H} 5$ and $\mathrm{H} 6$ and analogy with compound $\mathbf{4 b}$. Spectra showed a mixture of two conformers $(75 \%(\alpha): 25 \%(\beta))$.

IR: $3433,2983,2925,1707 \mathrm{~cm}^{-1}$.

${ }^{1} \mathrm{H}$ NMR (400 MHz, $\left.\mathrm{CDCl}_{3}\right) \delta=7.40-7.29(\mathrm{~m}, 5 \mathrm{H}, \mathrm{CH} \mathrm{Ar}), 5.26-5.10(\mathrm{~m}$, $\left.2 \mathrm{H}, \mathrm{CH}_{2} \mathrm{Ar}\right), 4.21-4.00\left(\mathrm{~m}, 5 \mathrm{H}, \mathrm{CH}_{2} \mathrm{CH}_{3}+\mathrm{H} 3+\mathrm{H} 4+\mathrm{H} 5\right), 3.54(\mathrm{dd}, J=8.4$, $2.8 \mathrm{~Hz}, 1 \mathrm{H}, \mathrm{H} 1$, conformer $\beta$ ), $3.48(\mathrm{dd}, J=8.5,2.6 \mathrm{~Hz}, 1 \mathrm{H}, \mathrm{H} 1$, conformer $\alpha), 2.99(\mathrm{~d}, J=13.9 \mathrm{~Hz}, 1 \mathrm{H}, \mathrm{H} 3$, conformer $\beta$ ), $2.89(\mathrm{~d}, J=14.0 \mathrm{~Hz}, 1 \mathrm{H}, \mathrm{H} 3$, conformer $\alpha$ ), 2.39 (dd, $J=5.7,2.6 \mathrm{~Hz}, 1 \mathrm{H}, \mathrm{H} 7), 2.16-2.09(\mathrm{~m}, 1 \mathrm{H}, \mathrm{H} 6)$, $1.29-1.25\left(\mathrm{~m}, 3 \mathrm{H}, \mathrm{CH}_{2} \mathrm{CH}_{3}\right.$, conformer $\left.\beta\right), 1.19\left(\mathrm{t}, J=7.2 \mathrm{~Hz}, 3 \mathrm{H}, \mathrm{CH}_{2} \mathrm{CH}_{3}\right.$, conformer $\alpha$ ).

${ }^{13} \mathrm{C}$ NMR $\left(101 \mathrm{MHz}, \mathrm{CDCl}_{3}\right.$, data of major conformer) $\delta=171.5\left(\mathrm{CO}_{2}\right)$, 157.4 $\left(\mathrm{NCO}_{2}\right), 136.4(\mathrm{C} \mathrm{Ar}), 128.4(\mathrm{CH} \mathrm{Ar}), 127.9$ ( $\left.\mathrm{CH} \mathrm{Ar}\right), 127.4(\mathrm{CH} \mathrm{Ar})$, 67.8 (C4), $67.6\left(\mathrm{CH}_{2}-\mathrm{Ar}\right), 64.3$ (C5), $60.8\left(\mathrm{CH}_{2} \mathrm{CH}_{3}\right), 46.3$ (C3), 39.8 (C1), 26.8 (C6), 26.5 (C7), $14.1\left(\mathrm{CH}_{2} \mathrm{CH}_{3}\right)$.

Anal. Calcd. for $\mathrm{C}_{17} \mathrm{H}_{21} \mathrm{NO}_{6}(335.35 \mathrm{~g} / \mathrm{mol}): \mathrm{C}, 60.9$; H, $6.3 \%$. Found: $\mathrm{C}$, 61.0; $\mathrm{H}, 6.6 \%$.

$\left(1 S^{*}, 4 S^{*}, 5 R^{*}, 6 R^{*}, 7 R^{*}\right)$-Ethyl 4,5-dihydroxy-7-(hydroxymethyl)-2azabicyclo[4.1.0]heptane-2-carboxylate, 6a: To a solution of $150 \mathrm{mg}$ $(0.55 \mathrm{mmol})$ of $\mathbf{4 a}$ in $2.2 \mathrm{~mL}$ of THF, $36 \mathrm{mg}(1.65 \mathrm{mmol})$ of $\mathrm{LiBH}_{4}$ was added in portions at $0^{\circ} \mathrm{C}$ and the resulting mixture was stirred at room temperature overnight. Then, acid amberlite was added, the mixture diluted with $\mathrm{MeOH}$ and filtrated to give $89 \mathrm{mg}(0.38 \mathrm{mmol}, 71 \%$ yield, $\mathrm{Rf}=0.1$ in EtOAc) of pure $\mathbf{6 a}$ as a colorless oil. Spectra showed a mixture of two conformers $(60 \%(\alpha): 40 \%(\beta))$.

IR: $3398,3022,2932,1680 \mathrm{~cm}^{-1}$

${ }^{1} \mathrm{H}$ NMR (400 MHz, CD $\left.{ }_{3} \mathrm{OD}\right) \delta=4.24-4.09\left(\mathrm{~m}, 3 \mathrm{H}, \mathrm{CH}_{2} \mathrm{CH}_{3}+\mathrm{H} 5\right), 3.63$ (dd, $J=11.5,5.8 \mathrm{~Hz}, 1 \mathrm{H}, \mathrm{CH} H \mathrm{OH}), 3.58(\mathrm{dd}, J=12.2,3.8 \mathrm{~Hz}, 1 \mathrm{H}, \mathrm{H} 3$, conformer $\alpha$ ), 3.52 (dd, $J=12.1,4.0 \mathrm{~Hz}, 1 \mathrm{H}, \mathrm{H} 3$, conformer $\beta$ ), $3.46-3.37$ (m, 1H, H4), $3.37-3.28(\mathrm{~m}, 1 \mathrm{H}, \mathrm{CHHOH}), 3.02(\mathrm{dd}, J=12.1,10.4 \mathrm{~Hz}, 1 \mathrm{H}$, 
$\mathrm{H} 3$, conformer $\beta$ ), $2.93(\mathrm{dd}, J=12.2,10.6 \mathrm{~Hz}, 1 \mathrm{H}, \mathrm{H} 3$, conformer $\alpha$ ), 2.80 $2.74(\mathrm{~m}, 1 \mathrm{H}, \mathrm{H} 1), 1.41(\mathrm{~m}, 1 \mathrm{H}, \mathrm{H} 6) 1.31-1.25\left(\mathrm{~m}, 3 \mathrm{H}, \mathrm{CH}_{2} \mathrm{CH}_{3}\right), 0.91$ $0.84(\mathrm{~m}, 1 \mathrm{H}, \mathrm{H} 7)$.

${ }^{13} \mathrm{C}$ NMR (101 MHz, CD $\left.{ }_{3} \mathrm{OD}\right) \delta=158.9\left(\mathrm{CO}_{2}\right.$, conformer $\left.\alpha\right), 158.5\left(\mathrm{CO}_{2}\right.$, conformer $\beta$ ), 68.6 (C4, conformer $\alpha$ ), 68.4 ( $\mathrm{C} 4$, conformer $\beta$ ), 66.7 (C5 conformer $\alpha$ ), 66.5 ( $\mathrm{C} 5$, conformer $\beta$ ), $64.0\left(\mathrm{CH}_{2} \mathrm{OH}\right.$, conformer $\left.\beta\right), 63.7$ $\left(\mathrm{CH}_{2} \mathrm{OH}\right.$, conformer $\left.\alpha\right)$, $62.9\left(\mathrm{CH}_{2} \mathrm{CH}_{3}\right.$, conformer $\left.\beta\right), 62.8\left(\mathrm{CH}_{2} \mathrm{CH}_{3}\right.$, conformer $\alpha$ ), 42.9 (C3, conformer $\beta$ ), 42.3 (C3, conformer $\alpha$ ), 34.0 (C1, conformer $\beta$ ), 33.2 (C1, conformer $\alpha$ ), 27.5 (C7, conformer $\alpha$ ), 27.1 (C7, conformer $\beta$ ), $25.5(\mathrm{C} 6$, conformer $\alpha$ ), 24.9 (C6, conformer $\beta$ ), 15.0 $\left(\mathrm{CH}_{2} \mathrm{CH}_{3}\right.$, conformer $\left.\alpha\right), 14.9\left(\mathrm{CH}_{2} \mathrm{CH}_{3}\right.$, conformer $\left.\beta\right)$.

Anal. Calcd. for $\mathrm{C}_{10} \mathrm{H}_{17} \mathrm{NO}_{5}$ (231.25 g/mol): C, 51.9; H, 7.4 \%. Found: C, 52.2; H, $7.2 \%$.

$\left(1 S^{*}, 4 R^{*}, 5 S^{*}, 6 R^{*}, 7 R^{*}\right)$-Ethyl 4,5-dihydroxy-7-(hydroxymethyl)-2azabicyclo[4.1.0]heptane-2-carboxylate, 6b: Following the procedure for the synthesis of $\mathbf{6 a}$, from $130 \mathrm{mg}(0.48 \mathrm{mmol}$ ) of $\mathbf{4 b}$ and $31 \mathrm{mg}$ ( 1.43 mmol) of $\mathrm{LiBH}_{4}, 75 \mathrm{mg}(0.32 \mathrm{mmol}, 67 \%$ of yield, $\mathrm{Rf}=0.3$ in EtOAc/EtOH (9:1)) of pure $\mathbf{4 b}$ were obtained as a colorless oil. Spectra showed a mixture of two conformers $(71 \%(\alpha): 29 \%(\beta))$.

IR: $3391,3020,2936,1685 \mathrm{~cm}^{-1}$.

${ }^{1} \mathrm{H}$ NMR (400 MHz, $\left.\mathrm{CD}_{3} \mathrm{OD}\right) \delta=4.21-4.09\left(\mathrm{~m}, 2 \mathrm{H}, \mathrm{CH}_{2} \mathrm{CH}_{3}\right), 4.05$ (dd, $J=$ 6.6, $4.5 \mathrm{~Hz}, 1 \mathrm{H}, \mathrm{H} 5), 3.96-3.81(\mathrm{~m}, 2 \mathrm{H}, \mathrm{H} 4+\mathrm{H} 3), 3.61(\mathrm{dd}, J=11.3,5.9$ $\mathrm{Hz}, 1 \mathrm{H}, \mathrm{CH} H \mathrm{OH}$, conformer $\alpha$ ), 3.54 (dd, $J=11.3,6.5 \mathrm{~Hz}, 1 \mathrm{H}, \mathrm{CH} H \mathrm{OH}$, conformer $\beta$ ), $3.39-3.26(\mathrm{~m}, 1 \mathrm{H}, \mathrm{CHHOH}), 2.93(\mathrm{~d}, J=12.8 \mathrm{~Hz}, 1 \mathrm{H}, \mathrm{H} 3$, conformer $\beta$ ), $2.84(\mathrm{dd}, J=13.4,1.5 \mathrm{~Hz}, 1 \mathrm{H}, \mathrm{H} 3$, conformer $\alpha$ ), 2.81 (dd, $J$ $=8.4,3.1 \mathrm{~Hz}, 1 \mathrm{H}, \mathrm{H} 1$, conformer $\alpha), 2.78(\mathrm{dd}, J=8.5,3.0 \mathrm{~Hz}, 1 \mathrm{H}, \mathrm{H} 1$, conformer $\beta$ ), $1.80-1.72(\mathrm{~m}, 1 \mathrm{H}, \mathrm{H} 7), 1.40-1.23\left(\mathrm{~m}, 4 \mathrm{H}, \mathrm{H} 6+\mathrm{CH}_{2} \mathrm{CH}_{3}\right.$ ).

${ }^{13} \mathrm{C}$ NMR (101 MHz, $\left.\mathrm{CD}_{3} \mathrm{OD}\right) \delta=159.8\left(\mathrm{CO}_{2}\right.$, conformer $\left.\alpha\right), 159.5\left(\mathrm{CO}_{2}\right.$, conformer $\beta$ ), 69.3 (C4, conformer $\alpha$ ), 69.2 (C4, conformer $\beta$ ), 66.3 (C5, conformer $\alpha$ ), 66.1 (C5, conformer $\beta$ ), $64.5\left(\mathrm{CH}_{2} \mathrm{OH}\right.$, conformer $\beta$ ), 64.3 $\left(\mathrm{CH}_{2} \mathrm{OH}\right.$, conformer $\left.\alpha\right)$, $62.8\left(\mathrm{CH}_{2} \mathrm{CH}_{3}\right.$, conformer $\left.\beta\right), 62.7\left(\mathrm{CH}_{2} \mathrm{CH}_{3}\right.$, conformer $\alpha$ ), 47.5 (C3, conformer $\beta$ ), 47.3 (C3, conformer $\alpha$ ), 36.6 (C1, conformer $\beta$ ), 35.7 (C1, conformer $\alpha$ ), 28.2 (C7, conformer $\alpha$ ), 27.8 (C7, conformer $\beta$ ), 22.9 (C6, conformer $\alpha$ ), 22.7 (C6, conformer $\beta$ ), 15.0 $\left(\mathrm{CH}_{2} \mathrm{CH}_{3}\right.$, conformer $\left.\alpha\right), 14.9\left(\mathrm{CH}_{2} \mathrm{CH}_{3}\right.$, conformer $\left.\beta\right)$.

Anal. Calcd. for $\mathrm{C}_{10} \mathrm{H}_{17} \mathrm{NO}_{5}$ (231.25 g/mol): C, 51.9; H, $7.4 \%$. Found: $\mathrm{C}$ $52.1 ; \mathrm{H}, 7.8 \%$.

$\left(1 S^{*}, 4 S^{*}, 5 R^{*}, 6 R^{*}, 7 R^{*}\right)$-Benzyl 4,5-dihydroxy-7-(hydroxymethyl)-2 azabicyclo[4.1.0]heptane-2-carboxylate, 7a: Following the procedure for the synthesis of $\mathbf{6 a}$, from $91 \mathrm{mg}(0.28 \mathrm{mmol})$ of $\mathbf{5 a}$ and $17.7 \mathrm{mg}(0.84$ mmol) of $\mathrm{LiBH}_{4}$ and after flash column chromatography in Hexane/EtOAc (1:19) $58 \mathrm{mg}(0.20 \mathrm{mmol}, 72 \%$ yield, $\mathrm{Rf}=0.26$ in Hexane/EtOAc, 1:4) of $\mathbf{7 a}$ were isolated as a colorless oil. Spectra showed a mixture of two conformers $(55 \%(\alpha): 45 \%(\beta))$

IR: $3400,3025,2933,1684 \mathrm{~cm}^{-1}$

${ }^{1} \mathrm{H}$ NMR (400 MHz, $\left.\mathrm{CDCl}_{3}\right) \delta=7.36-7.28(\mathrm{~m}, 5 \mathrm{H}, \mathrm{CH} \mathrm{Ar}), 5.17-5.04(\mathrm{~m}$, $2 \mathrm{H}, \mathrm{CH}_{2}$-Ar), $4.24-4.15(\mathrm{~m}, 1 \mathrm{H}, \mathrm{H} 5), 3.70-3.60(\mathrm{~m}, 1 \mathrm{H}, \mathrm{CH} \mathrm{HOH}), 3.67$ (dd, $J=12.0,4.3 \mathrm{~Hz}, 1 \mathrm{H}, \mathrm{H} 3$, conformer $\alpha$ ), $3.58(\mathrm{dd}, J=12.4,3.9 \mathrm{~Hz}, 1 \mathrm{H}$, $\mathrm{H} 3$, conformer $\beta$ ), $3.48-3.42(\mathrm{~m}, 1 \mathrm{H}, \mathrm{H} 4), 3.36(\mathrm{dd}, J=6.9,2.8 \mathrm{~Hz}, 1 \mathrm{H}$ $\mathrm{CH} H \mathrm{OH}$, conformer $\alpha), 3.30-3.21(\mathrm{~m}, 1 \mathrm{H}, \mathrm{CH} H \mathrm{OH}$, conformer $\beta), 3.02(\mathrm{t}$ $J=11.5 \mathrm{~Hz}, 1 \mathrm{H}, \mathrm{H} 3$, conformer $\beta), 2.95(\mathrm{t}, J=11.5 \mathrm{~Hz}, 1 \mathrm{H}, \mathrm{H} 3$, conformer $\alpha), 2.77(\mathrm{dd}, J=8.1,2.9 \mathrm{~Hz}, 1 \mathrm{H}, \mathrm{H} 1$, conformer $\beta), 2.73(\mathrm{dd}, J=8.0,2.8 \mathrm{~Hz}$, $1 \mathrm{H}, \mathrm{H} 1$, conformer $\alpha), 1.41(\mathrm{~m}, 1 \mathrm{H}, \mathrm{H} 6), 0.90-0.80$ (m, 1H, H7).

${ }^{13} \mathrm{C}$ NMR (101 MHz, $\left.\mathrm{CDCl}_{3}\right) \delta=156.9\left(\mathrm{NCO}_{2}\right.$, conformer $\left.\beta\right), 156.7\left(\mathrm{NCO}_{2}\right.$, conformer $\alpha$ ), 136.0 (C Ar, conformer $\beta$ ), 135.9 (C Ar, conformer $\alpha$ ), 128.7 ( $\mathrm{CH} \mathrm{Ar}$ ), 128.5 ( $\mathrm{CH} \mathrm{Ar}$ ), 128.4 ( $\mathrm{CH} \mathrm{Ar}), 128.2$ ( $\mathrm{CH} \mathrm{Ar}), 128.0$ ( $\mathrm{CH} \mathrm{Ar}$ ), 127.9( $\mathrm{CH} \mathrm{Ar}), 67.8\left(\mathrm{CH}_{2}-\mathrm{Ar}\right.$, conformer $\left.\alpha\right), 67.6\left(\mathrm{CH}_{2}-\mathrm{Ar}\right.$, conformer $\left.\beta\right)$, $67.5(\mathrm{C} 4$, conformer $\beta$ ), $67.4(\mathrm{C} 4$, conformer $\alpha$ ), $65.3(\mathrm{C} 5$, conformer $\beta$ ), $65.3\left(\mathrm{C} 5\right.$, conformer $\alpha$ ), $63.6\left(\mathrm{CH}_{2} \mathrm{OH}\right.$, conformer $\left.\alpha\right), 63.3\left(\mathrm{CH}_{2} \mathrm{OH}\right.$, conformer $\beta$ ), 41.8 (C3, conformer $\beta$ ), 41.2 (C3, conformer $\alpha$ ), 33.1 (C1, conformer $\beta$ ), 32.3 (C1, conformer $\alpha$ ), 26.6 (C7, conformer $\alpha$ ), 26.4 (C7, conformer $\beta$ ), 24.0 (C6, conformer $\beta$ ), 23.4 (C6, conformer $\alpha$ ).

Anal. Calcd. for $\mathrm{C}_{17} \mathrm{H}_{21} \mathrm{NO}_{6}(293.32 \mathrm{~g} / \mathrm{mol})$ : C, 61.4; H, $6.5 \%$. Found: $\mathrm{C}$, $61.2 ; \mathrm{H}, 6.3 \%$. $\left(1 S^{*}, 4 R^{*}, 5 S^{*}, 6 R^{*}, 7 R^{*}\right)$-Benzyl 4,5-dihydroxy-7-(hydroxymethyl)-2azabicyclo[4.1.0]heptane-2-carboxylate, 7b: Following the procedure for the synthesis of $\mathbf{6 a}$, from $113 \mathrm{mg}(0.35 \mathrm{mmol})$ of $\mathbf{5 b}$ and $22.0 \mathrm{mg}$ of $\mathrm{LiBH}_{4}$ and after flash column chromatography in Hexane/EtOAc (1:19) $80 \mathrm{mg}(0.28 \mathrm{mmol}, 80 \%$ yield, $\mathrm{Rf}=0.3$ in EtOAc/EtOH (9:1)) pure $\mathbf{7 b}$ were isolated as a colorless oil. Spectra showed a mixture of two conformers $(60 \%(\alpha): 40 \%(\beta))$.

IR: 3395, 3022, 2939, $1690 \mathrm{~cm}^{-1}$.

${ }^{1} \mathrm{H}$ NMR (400 MHz, $\left.\mathrm{CDCl}_{3}\right) \delta 7.36-7.27(\mathrm{~m}, 5 \mathrm{H}, \mathrm{CH} \mathrm{Ar}), 5.24-4.98(\mathrm{~m}$, $2 \mathrm{H}, \mathrm{CH}_{2}$-Ar), $4.10-3.96(\mathrm{~m}, 2 \mathrm{H}, \mathrm{H} 3+\mathrm{H} 5), 3.95-3.90(\mathrm{~m}, 1 \mathrm{H}, \mathrm{H} 4$, conformer $\alpha$ ), $3.89-3.84(\mathrm{~m}, 1 \mathrm{H}, \mathrm{H} 4$, conformer $\beta$ ), $3.70(\mathrm{dd}, J=11.3,5.6$ $\mathrm{Hz}, 1 \mathrm{H}, \mathrm{CH} H \mathrm{OH}$, conformer $\alpha$ ), $3.59(\mathrm{dd}, J=11.3,6.8 \mathrm{~Hz}, 1 \mathrm{H}, \mathrm{CH} H \mathrm{OH}$, conformer $\beta$ ), $3.30(\mathrm{dd}, J=11.3,7.4 \mathrm{~Hz}, 1 \mathrm{H}, \mathrm{CH} H \mathrm{OH}$, conformer $\beta$ ), 2.95 (dd, $J=11.3,9.0 \mathrm{~Hz}, 1 \mathrm{H}, \mathrm{CH} H \mathrm{OH}$, conformer $\alpha), 2.88-2.69(\mathrm{~m}, 2 \mathrm{H}, \mathrm{H} 3+$ H1), 1.87 - 1.77 (m, 1H, H7), $1.41-1.26$ (m, 1H, H6).

${ }^{13} \mathrm{C}$ NMR (101 MHz, $\left.\mathrm{CDCl}_{3}\right) \delta 157.9\left(\mathrm{NCO}_{2}\right.$, conformer $\left.\alpha\right), 157.6\left(\mathrm{NCO}_{2}\right.$, conformer $\beta$ ), 136.4 (C Ar, conformer $\beta$ ), 136.3 (C Ar, conformer $\alpha$ ), 128.5 ( $\mathrm{CH}$ Ar conformer $\alpha$ ), 128.4 (CH Ar, conformer $\beta$ ), 128.1 ( $\mathrm{CH} \mathrm{Ar}$, conformer $\alpha$ ), 128.0 ( $\mathrm{CH} \mathrm{Ar}$, conformer $\beta$ ), 127.7 (CH Ar), 67.8 (C4), 67.6 $\left(\mathrm{CH}_{2}-\mathrm{Ar}\right.$, conformer $\left.\alpha\right), 67.3\left(\mathrm{CH}_{2}-\mathrm{Ar}\right.$, conformer $\beta$ ), 65.1 (C5, conformer $\alpha$ ), 65.0 (C5, conformer $\beta$ ), $64.2\left(\mathrm{CH}_{2} \mathrm{OH}\right), 46.4$ (C3, conformer $\alpha$ ), 46.3 (C3, conformer $\beta$ ), 36.1 (C1, conformer $\beta$ ), 35.1 (C1, conformer $\alpha$ ), 27.7 (C7, conformer $\alpha$ ), 27.2 (C7, conformer $\beta$ ), 22.5 (C6, conformer $\alpha$ ), 22.0 (C6, conformer $\beta$ ).

Anal. Calcd. for $\mathrm{C}_{15} \mathrm{H}_{19} \mathrm{NO}_{5}$ (293.32 g/mol): C, 61.4; H, 6.5 \%. Found: C, 61.7; H, $6.2 \%$.

\section{Funding Information}

Funding of this project by Spanish MINECO and Fondo Europeo de Desarrollo Regional (FEDER, grant No. CTQ2015-64624-R MINECO/FEDER) and FUSP-CEU (PC17/16) is acknowledged.

\section{Acknowledgment}

A. L-R. thanks the Fundación San Pablo-CEU for a pre-doctoral fellowship

\section{Supporting Information}

YES (this text will be updated with links prior to publication)

\section{Primary Data}

NO (this text will be deleted prior to publication)

\section{References}

(1) (a) Rempel, B. P.; Withers, S. G. Glycobiology 2008, 18, 570. (b) Asano, N. Glycobiology 2003, 13, 93R.

(2) For biological activity of iminosugars, see: (a) Tyrrell, B. E.; Sayce, A. C.; Warfield, K. L.; Miller, J. L.; Zitzmann, N. Crit. Rev. Microbiol. 2017, 43, 521. (b) Horne G.; Wilson, F. X. Prog. Med. Chem. 2011, 50, 135. (c) Nash, R. J.; Kato, A.; Yu C.-Y.; Fleet, G. W. Future Med. Chem. 2011, 3, 1513. (d) Wrodnigg, T. M.; Steiner, A. J.; Ueberbacher, B. J. Anti-Cancer Agents Med. Chem. 2008, 8, 77. (e) Gerber-Lemaire, S.; Juillerat-Jeanneret, L. Mini-Rev. Med. Chem. 2006, 6, 1043. (f) Robina, I.; Moreno-Vargas, A. J.; Carmona, A. T.; Vogel, P. Curr. Drug Metab. 2004, 5, 329. (g) Lillelund, V. H.; Jensen, H. H.; Liang X.; Bols, M. Chem. Rev. 2002, 102, 515.

(3) Compain, P.; Martin, O. R. Iminosugars-from synthesis to therapeutical applications; John Wiley \&Sons: Chichester, U.K. 2008. (b) Stütz, A. E. Iminosugars as Glycosidase InhibitorsNojirimycin and beyond, Wiley-VCH, 1999. (c) Butters, T. D.; Dwek, R. A.; Platt, F. M. Chem.Rev. 2000, 100, 4683.

(4) Joubert, P. H.; Veuter, C. P.; Joubert H. F.; Hillebrand, I. Eur. J. Clin. Pharmacol. 1985, 28, 705. Review: Winchester, B. G. Tetrahedron: Asymm. 2009, 20, 645.

(5) (a) Yoshikuni, Y.; Ezure, Y.; Seto, T.; Mori, K.; Watanabe M.; Enomoto, H. Chem. Pharm. Bull. 1989, 37, 106. (b) Markad, S. D.; 
Karanjule, N. S.; Sharma, T.; Sabharwal S. G.; Dhavele, D. D. Bioorg. Med. Chem., 2006, 14, 5535.

(6) Reviews, see: (a) Winchester, B. G. Tetrahedron: Asymm. 2009, 20 645. (b) Afarinkia, K.; Bahar, A. Tetrahedron: Asymm. 2005, 16 1239. (c) Pearson, M. S. M.; Mathé-Allainmat, M.; Fargeas, V.; Lebreton, J. Eur. J. Org. Chem. 2005, 2159. (d) Ayad, T.; Genisson, Y.; Baltas, M. Curr. Org. Chem. 2004, 8, 1211. (e) Weintraub, P. M.; Sabol, J. S.; Kane, J. M.; Borcherding, D. R. Tetrahedron 2003, 59, 2953.

(7) (a) Niwa, T.; Tsuruoka, T.; Goi, H.; Kodama, Y.; Itoh, J.; Inouye, S.; Yamada, Y.; Niida, T.; Nobe M.; Ogawa, Y. J. Antibiot. 1984, 37, 1579. (b) Inouye, S.; Tsuroka T.; Niida, T. J. Antibiot. Ser. A 1966, XIX, 288.

(8) Selected recent references: (a) Viuff, A. H.; Besenbacher, L. M.; Kamori, A.; Jensen, M. T.; Kilian, M.; Kato, A.; Jensen, H. H.; Org Biomol. Chem. 2015, 13, 9637. (b) Ganesan, M.; Salunke, R. V.; Singh, N.; Ramesh, N. G. Org. Biomol. Chem. 2013, 11, 599. (c) Singh, A.; Kim, B.; Lee, W. K.; Ha, H.-J. Org. Biomol. Chem. 2011, 9 1372. (d) Tamayo, J. A.; Franco F.; Re, D. L. Synlett 2010, 1323. (e) Karjalainen, O. K.; Passiniemi M.; Koskinen, A. M. P. Org. Lett 2010, 12, 1145. (f) Bagal, S. K.; Davies, S. G.; Lee, J. A.; Roberts, P. M.; Russell, A. J.; Scott P. M.; Thomson, J. E. Org. Lett. 2010, 12, 136. (g) Guaragna, A.; D’Errico, S.; D’Alonzo, D.; Pedatella, S.; Palumbo, G. Org. Biomol. Chem. 2010, 8, 3307. (h) van den Nieuwendijk, A. M. C. H.; Ruben, M.; Engelsma, S. E.; Risseeuw, M. D. P.; van den Berg, R. J. B. H. N.; Boot, R. G.; Aerts, J. M.; Brussee, J.; van der Marel, G. A.; Overkleeft, H. S. Org. Lett. 2010, 12, 3857. (i) Aravind, A.; Sankar, M. G.; Varghese, B.; Baskaran, S. J. Org. Chem. 2009, 74, 2858. (j) Palyam N.; Majewski, M. J. Org. Chem. 2009, 74, 4390. (k) Rengasamy, R.; Curtis-Long, M. J.; Ryu, H. W.; Oh K. Y.; Park, K. H. Bull. Korean Chem. Soc. 2009, 30, 1531. (1) Fu, R.; Du, Y.; Li, Z.-Y.; Xu W.-X.; Huang, P.-Q. Tetrahedron 2009, 65, 9765. (m) Rengasamy, R.; Curtis-Long, M. J.; Seo, W. D.; Jeong, S. H.; Jeong, I.-Y.; Park, K. H. J. Org. Chem. 2008, 73, 2898. (n) Kumar, A.;
Rawal, G. K.; Vankar, Y. D. Tetrahedron 2008, 64, 2379. (o) Sanap S. P.; Ghosh, S.; Jabgunde, A. M.; Pinjari, R. V.; Gejji, S. P.; Singh, S.; Chopade, B. A.; Dhavale, D. D. Org. Lett. 2007, 9, 3473. (p) Martín, R.; Murruzzu, C.; Pericàs, M. A.; Riera, A. J. Org. Chem. 2005, 70, 3326. (q) Takahata, H.; Banba, Y.; Ouchi, H.; Nemoto, H. Org. Lett. 2003, 5, 2527.

(9) For a review on conformationally restricted glycoside derivatives, see: Maaliki, C.; Gauthier, C.; Massinon, O.; Sagar, R.; Vincent, S. P.; Blériot, Y. Carbohydr. Chem. 2014, 40, 418.

(10) Desire, J.; Shipman, M. Synlett 2001, 1332. For an aziridine containing iminosugar derivative, see: Merino, P.; Tejero, T.; Laguna, M.; Cerrada, E.; Moreno, A.; Lopez, J. A. Org. Biomol. Chem 2003, 1, 2336.

(11) (a) Sánchez-Fernández, E. M.; Gonçalves-Pereira, R.; RísquezCuadro, R.; Plata, G. B.; Padrón, J. M.; García Fernández, J. M.; Ortiz Mellet, C. Carbohydr. Res. 2016, 429, 113. (b) Li, Y.-X. Shimada, Y.; Adachi, I.; Kato, A.; Jia, Y.-M.; Fleet, G. W. J.; Xiao, M.; Yu, C.-Y. J. Org. Chem. 2015, 80, 5151. (c) Cipolla, L.; Fernandes, M. R.; Gregori, M.; Airoldi, C.; Nicotra, F. Carbohydr. Res. 2007, 342, 1813.

(12) (a) Barbachyn, M. R.; Ford, C. W. Angew. Chem., Int. Ed. 2003, 42, 2010. (b) Schierle-Arndt, K.; Kolter, D.; Danielmeier, K.; Steckhan, E. Eur. J. Org. Chem. 2001, 2425. (c) Zurenko, G. E.; Gibson, J. K.; Shinabarger, D. L.; Aristoff, P. A.; Ford, C. W.; Tarpley, W. G. Curr. Opin. Pharmacol. 2001, 1, 470.

(13) (a) Niphakis, M. J.; Turunen, B. J.; Georg, G. I. J. Org. Chem. 2010, 75, 6793. (b) Ege, M. Wanner, K. T. Org. Lett. 2004, 6, 3553. (c) Ma, D.; Sun, H. Org. Lett. 2000, 2, 2503.

(14) Sebesta, R.; Pizzuti, M. G.; Boersma, A. J.; Minnaard, A, J.; Feringa, B. L. Chem. Commun. 2005, 1711.

(15) López-Rodríguez, A.; Domínguez, G.; Pérez-Castells. J.; ChemistrySelect 2017, 2, 2565.

\section{Biosketches}

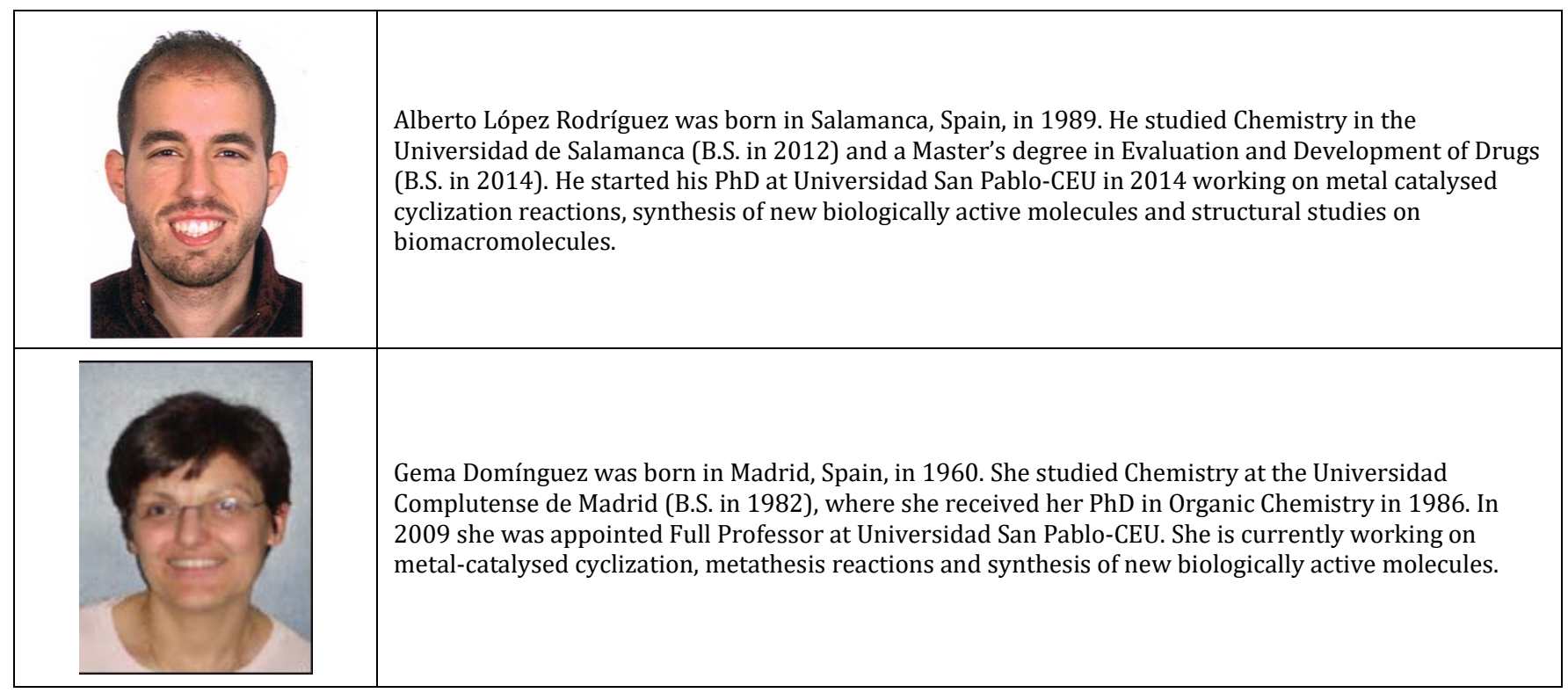




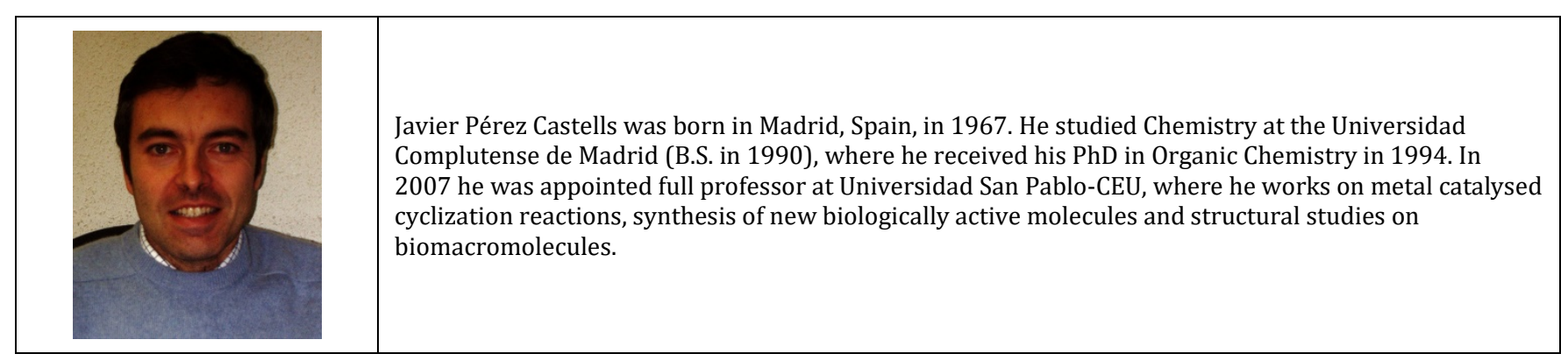

